\title{
Proceeding
}

Supplementary Issue: Summer Conferences of Sports Science. Costa Blanca Sports Science Events, 25-26 September 2020. Alicante, Spain.

\section{Comparison of EEG biofeedback and visual search strategies during e-sports play according to skill level}

\author{
RYOUSUKE FURUKADO ${ }^{1,3} \triangleleft$, GOICHI HAGIWARA2 ${ }^{2}$ TOMOHIRO ITO ${ }^{3}$, HIROHISA ISOGAI 2,3 \\ ${ }^{1}$ Integrated System Engineering, Faculty of Engineering, Nishinippon Institute of Technology, Fukuoka, Japan \\ ${ }^{2}$ Department of Sport Science and Health, Faculty of Human Science, Kyushu Sangyo University, Fukuoka, Japan \\ ${ }^{3}$ Fukuoka Sports Research Consortium, Fukuoka, Japan
}

\begin{abstract}
About $83 \%$ of the perceptual information humans receive from the outside world comes through the visual domain, making the tracking of visual information important for the superior performance of e-sports players. In addition, it is known that e-sports players' emotional state during performance affects their results. However, few studies have examined visual search strategies and electroencephalogram (EEG) findings of e-sports players while engaged in e-sports. Therefore, the present study aimed to investigate the characteristics of visual search activity and EEG feedback during first person shooter (FPS) game play, in which the tracking of visual information is important, and to examine the characteristics of different gaming skill levels. Four skilled and five semi-skilled e-sports participants (mean age $\pm 19.11, \mathrm{SD}=0.99$ ) participated in this study. Gazepoint GP3 (Canada) was used to measure eye movements, and the Sports KANSEI (Littlesoftware Inc., Japan) was used to analyse the emotional state of the participants by using EEG data. The results showed that the skilled e-sports players checked the camera significantly more frequently than the semi-skilled participants. The results of the area of interest (AOI) analysis showed that skilled e-sports players glanced at the friendly team information displayed at the top of the game screen more often than semi-skilled players. Furthermore, it was found that skilled e-sports players had significantly higher average EEG relaxation values during gameplay than semi-skilled players. Thus, the visual search strategy and emotional state while playing e-sports differed according to gaming skill level.

Keywords: Visual search strategy; Eye tracking; EEG; e-sports; FPS games.

\section{Cite this article as:}

Furukado, R., Hagiwara, G., Ito, T., \& Isogai, H. (2020). Comparison of EEG biofeedback and visual search strategies during e-sports play according to skill level. Journal of Human Sport and Exercise, 15(4proc), S1123S1132. doi:https://doi.org/10.14198/ihse.2020.15.Proc4.13

Corresponding author. Integrated System Engineering, Faculty of Engineering, Nishinippon Institute of Technology, 800-0394, Kanda-machi, Miyako-gun, Fukuoka, Japan.

E-mail: furukado@nishitech.ac.jp

Abstract submitted to: Spring Conferences of Sports Science. Costa Blanca Sports Science Events, 19-20 June 2020. Alicante, Spain.

JOURNAL OF HUMAN SPORT \& EXERCISE ISSN 1988-5202

(c) Faculty of Education. University of Alicante

doi:10.14198/jhse.2020.15.Proc4.13
\end{abstract}




\section{INTRODUCTION}

The e-sports market size is growing annually with numerous events taking place globally and is valued at $\$ 159.3$ billion in 2020. Many colleges and universities already have national e-sports teams, and some offer scholarships, similar to sports scholarships (Morrison, 2018). A wide variety of game genres exist in e-sports and can be broadly classified into 3 main categories: multiplayer online battle arena games (MOBA), roleplaying games, and shooting games (Bonny \& Castaneda, 2017).

In first-person shooter (FPS) games, targets are meant to move arbitrarily through space depending upon players' subjective points of view, and require constant adaptive actions based on stimulus information obtained from audio-visual sources and past personal experience. FPS games are characterized by a faster pace than MOBA games (Alloza et al., 2018), and are also highly competitive, with multiple players working together to achieve shared goals and perform their respective roles for their teams to win. For instance, players need to understand information about the maps used in the game, and the properties of the characters they control (each weapon's speed of movement and recoil control). This knowledge is to some extent, acquired naturally through one's experience of playing the game.

In addition, it is said that during gameplay, one's environment changes in real time, and faster decisionmaking, strategizing, and reaction times are, therefore, required to perform well in the game (Sousa et al., 2020). When confronted with an enemy player, quick and accurate decisions are important since players have less than a second to decide whether to fire or retreat, and this is called the decision-making ability. Various aspects of situational decision-making ability in elite athletes have been discussed in studies of expertise in open-skill sports, such as soccer and basketball. Kikumasa and Kokubu (2018) found that skilled individuals' unique visual search strategies contribute to good situational decision-making abilities. Furukado and Isogai (2020), too, pointed out the importance of visual search strategies that use empirical knowledge from the sporting context to predict what is likely to occur next in complex and dynamic sporting situations. Previous research, though limited in number, had also examined visual search strategies during FPS gameplay. Soderberg et al. (2012) examined the differences between visual search strategies adopted by military personnel and civilians during FPS gameplay and found that military personnel tended to pay less attention than civilians to the region of interest for tactical objects. To summarize the research to date, many studies have investigated visual search strategies in real sports such as soccer and basketball, but very few have investigated these strategies during FPS gameplay. When, where, and how to look, is considered important not only for FPS games but also for improving gaming skills.

Additionally, psychological skills are also important for the performance of e-sports. Hung et al. (2019) reported that during e-sports like gameplay, emotions affect players' performance. There is no arguing that in global tournaments, in which the future of players is decided with huge prizes at stake, there is a lot of psychological pressure on players. In support of this fact, previous studies have shown that during gameplay, heart rate increases to near sprint status (approximately 190-200) (Valladao et al., 2019). There are also psychologists, who currently provide mental training guidance to professional e-sports teams. If we can understand the players' specific emotional states during gameplay, we can also use this information for mental training guidance. Hagiwara, Kawahara, et al. (2020) examined electroencephalogram (EEG) measurements to understand emotional states during race gameplay. The results showed that the power percentages of the beta and sensorimotor rhythm (SMR) wave bands obtained from FP1 after race gameplay had significantly increased compared to the resting period. Campbell et al. (2018) suggested that e-sports genres should be distinguished with clear distinctions between them, in the same way as different traditional 
sports. In addition, little is known about effective training methods designed to optimize e-sports performance (Martin-Niedecken and Schättin, 2020).

Therefore, the present study aimed to investigate the characteristics of visual search activity and EEG feedback during FPS gameplay, in which the tracking of visual information is important for examining the characteristics of different gaming skill levels. Thus, examining e-sports players' superior performance by measuring their physiological indices during FPS gameplay may lead to the accumulation of knowledge for the development of research expertise. In addition, this study's results could be used to pilot a proposal for an efficient training method to solve the problem of game addiction.

\section{METHODS}

\section{Participants}

Nine male FPS players participated -5 skilled and 4 semi-skilled, mean (M) age of 19.11, standard deviation $(S D)=0.99$-who usually played games for at least 5 hours per day and 6 days a week. The grouping criteria were based on the match making rate (MMR) of "Tom Clancy's Rainbow Six: Siege" (Ubisoft, 2015), the FPS game used in this study. The outline and purpose of the study was explained to the participants, and their data and informed consent were obtained. All the participants had normal or corrected-to-normal vision.

\section{Apparatus}

The devices and environment used in the experiments are shown in figure 1 and table 1. We used the mouse, mouse pad, and keyboard that the participants generally used. Visual search strategies were measured using the Gazepoint GP3 HD eye tracker (Gazepoint, Canada). The sampling rate was $150 \mathrm{~Hz}$, and the minimum degree of resolution ranged from 0.5-1. EEG was measured using a device with a simple band-type EEG signal collection chip (Neuro Sky Co., Ltd, Japan), and the acquired data were recorded on a tablet device connected through Bluetooth. The acquired data were evaluated by using Sports KANSEI (Littlesoftware Inc., Japan), an application that outputs sensitivity values.

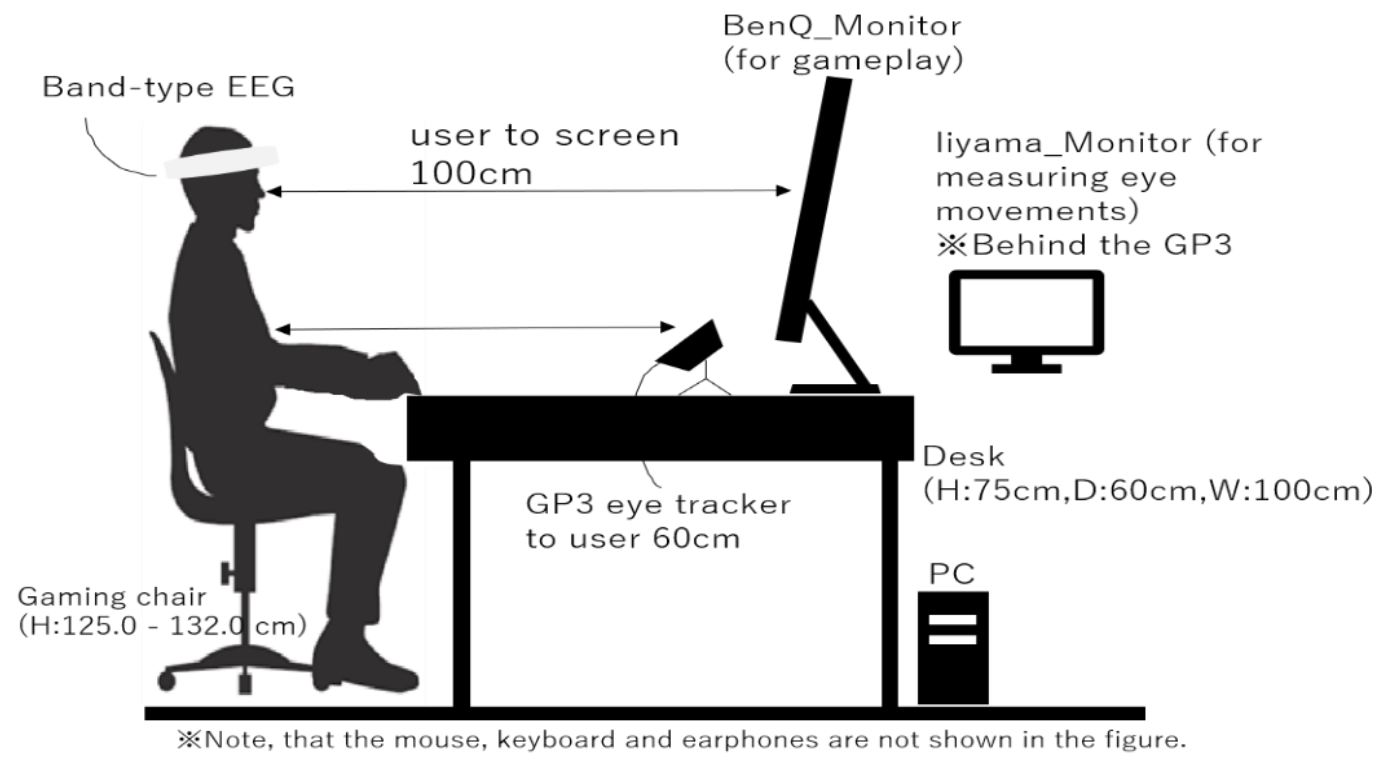

Figure 1 Equipment used in the experiment. 
Table 1. Detailed information on equipment used in the experiment.

\begin{tabular}{ll}
\hline Manufacturer & ThirdWave (THIRDWAVE Corp.) \\
\hline OS & Windows 10 Pro64bit \\
\hline Power supply (PSU) & Owltech AS 700 (700W/Gold+) \\
\hline Motherboard & ASUS H370-A-SI \\
\hline Processor (CPU) & Intel Core i7-9700K \\
\hline Graphics Card (GPU) & GeForce RTX 2080 \\
\hline Memory (RAM) & DDR4 16GB (8GB×2) \\
\hline Storage drive & W.D. M.2 SSD 500GB and TOSHIBA HDD 3TB (7200rpm) \\
\hline Cooling (CPU cooler) & DEEPCOOL / H-GAMMAXX400 \\
\hline Monitor & BenQ ZOWIE XL LCD (24 inch, refresh rate at 144Hz, for gameplay), \\
& iiyama XU2292HS-B1A (21.5 inch, for measure eyemovement) \\
\hline Earphones and Sound card & Sennheiser IE 40Pro(IE40PROBLACK) and Logicool Astro Mixamp Pro \\
(substitutes headset) & TR(MAPTR-002) \\
\hline
\end{tabular}

\section{Adopted game}

In this study, we used the Rainbow Six Siege, a 5-on-5 competitive FPS game, in which the participants, who were divided into attacker and defender teams, fought to wipe out enemy positions or defuse bombs in approximately 3 minutes per round. The number of rounds was set to two. In general, the balance of a game is determined by the MMR which reflects the real abilities of the opposing team and its friendly team in an informal match. In the present study, the experimental conditions were standardized by playing 5 versus 5 custom matches with the same subjects to prevent the replacement of enemy and friendly team members for each participant. In addition, to remove the influence of character differences, we made participants use the same operator, "Zofia" for attack and "Valkyrie" for defence. Furthermore, the map used in the experiment was the "Consulate". Voice chats were allowed only for listening to the voice, but vocalizations were not allowed. Participants were asked to play the game using settings, such as mouse sensitivity and key mapping, that they normally used when playing the game.

\section{Methods for measuring and assessing visual search strategies}

Eye movement data were used during gameplay to measure and assess the visual search strategy. Participants sat $100 \mathrm{~cm}$ away from the gameplay monitor and performed a 9-point calibration. A $60 \mathrm{~cm}$ distance was kept between the eye movement measurement device (GP3 HD) and the participants' eyes. The data were analysed only for the time that the participants were "alive" during the gameplay. This was because if a player controlled by the operator was defeated, the monitor would be viewed from another player's or surveillance camera's perspective that existed in the map, until the victory or defeat was determined.

This study conducted an area of interest (AOI) analysis to calculate the frequency of gazes in each area. The settings for $\mathrm{AOI}$ analysis were specified for each of the 5 fixed locations displayed on the game screen (Figure 2).

The gaze fixation time per gaze was calculated by dividing the total gaze time by the total number of gazes, based on the eye movement data during gameplay. In addition, the number of times the player looked at the surveillance cameras on the map (mainly used by the defenders) and the mobile drone cameras (used by the attackers), as well as the total number of times the players peeked at the gun's muzzle (the so-called Aim Down Sights) were calculated as FPS game-specific indicators. 


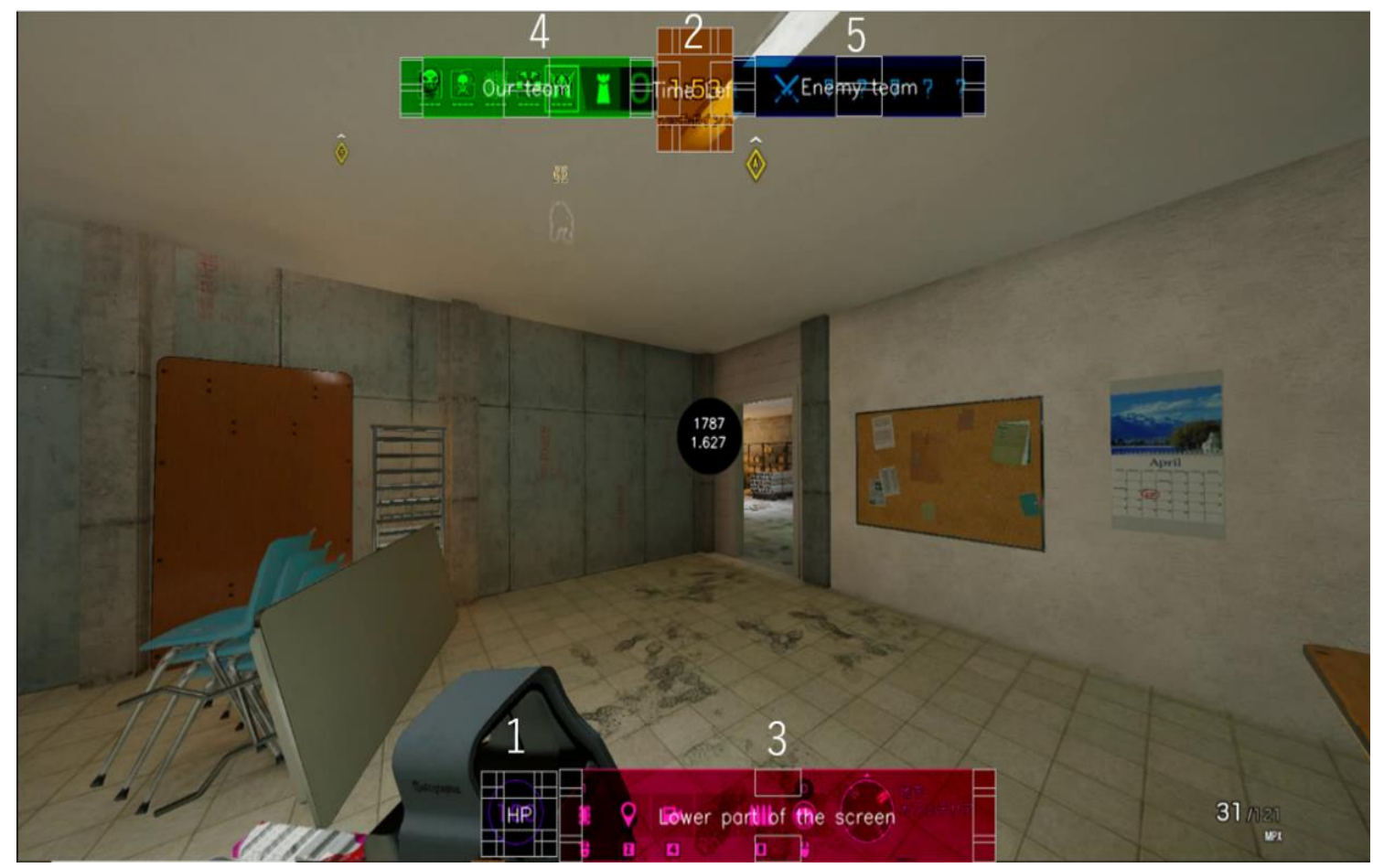

Figure 2. An analysis of the area of interest settings.

\section{Methods for measuring and assessing psychological states}

EEGs are electrical potentials generated on the scalp as a result of brain activity and are a part of biological information that continues to occur in humans (Miyata et al., 2004). The disadvantages of EEG include attenuation of the output signal and low spatial resolution. However, compared to functional magnetic resonance imaging, magnetoencephalography, and positron emission tomography; EEG does not require large equipment and is inexpensive. Thus, it has the advantage of being easy to use for research. The human psychological (mental) state can also be reflected by recording electrical signals obtained from electrodes placed on the scalp. For example, Hagiwara, Akiyama, et al. (2020) measured the EEG of FP1 (10-20 system) located in the left frontal lobe of participants performing the rhythm game and noted an increase in selective attention levels. In addition, Hagiwara et al. (2019) found that the concentration values during gameplay were significantly higher compared to the baseline, when the soccer game was performed for 10 minutes.

Therefore, in the present study, we used the same EEG measures as in the studies by Hagiwara et al. (2019); Hagiwara, Akiyama, et al. (2020). Specifically, the participants wore an EEG device on their heads, and electrodes were attached to their ears and forehead to obtain data on the intensity of alpha $(8-13 \mathrm{~Hz})$ and beta $(13-40 \mathrm{~Hz})$ waves, at 1-second intervals. The EEG data obtained in this way were recorded on a tablet device using Bluetooth communication, and analysed by the Sports KANSEI, which was finally output as a sensitivity value of $0-100$. It is possible to evaluate the three psychological states of concentration, relaxation, and zone (SMR wave) according to the size of the sensitivity values calculated by this algorithm (higher values indicate a higher degree of superiority). 


\section{Procedure}

The experimental procedure is shown in Figure 3. First, participants were required to wear an EEG device and underwent a 2-minute EEG measurement in an open-eyed state to determine their baseline level of arousal, prior to gameplay. They then performed a calibration for setting up the eye movement measurement device. After synchronizing the EEG and the eye movement device, gameplay was performed. These processes were repeated nine times in one cycle to measure the participants' data.

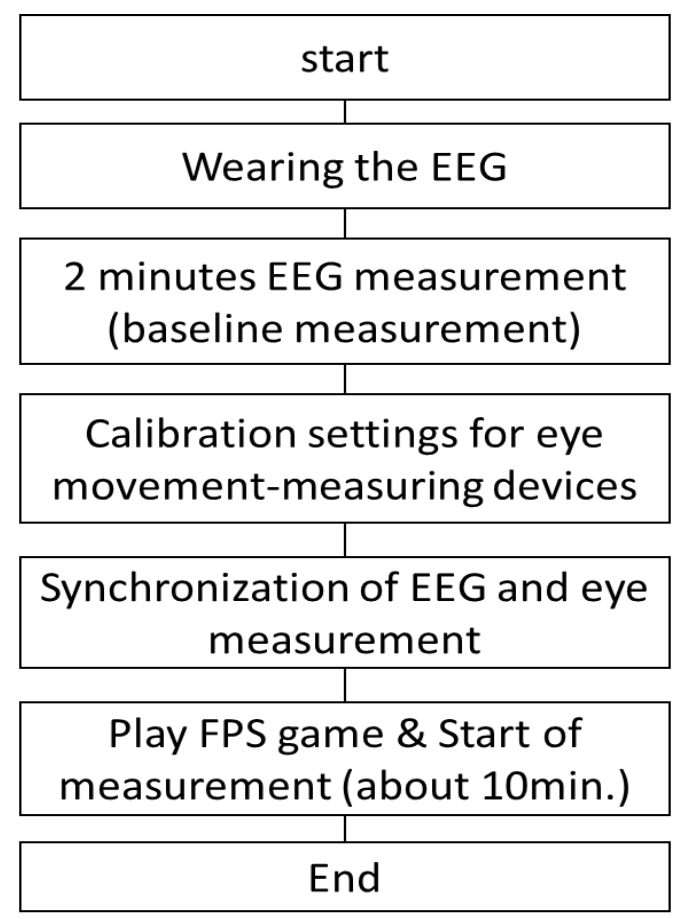

Figure 3. Procedure for the experiment.

\section{Analysis}

During gameplay, an unpaired t-test was conducted on the obtained eye movement data to characterize the visual search strategies of FPS players having different skill levels. In addition, a paired t-test was employed to compare the means of concentration, relaxation, and zone, during FPS gameplay with a 2-minute baseline measurement. Subsequently, an unpaired t-test was used to compare the means of concentration, relaxation, and zone during gameplay with different skill levels. IBM SPSS Statistics 27.0 software was used for all statistical analyses. A standardized measure was calculated of the effect size, which does not vary with sample size. The Cohen's d index (d) was used as the effect size. Gazepoint Analysis (Gazepoint, Canada) was also used to analyse eye movement data.

\section{RESULTS}

At the outset, we used eye movement data during FPS gameplay to examine the differences in visual search strategies between different skill levels. The AOI analysis revealed a significant difference in the number of times participants gazed at the "Our team" area during FPS games $(t(7)=2.97, p<.05, d=1.88)$. The skilled group ( $M=3.75, S D=2.87$ ) gazed at the "Our team" area significantly more often than the semi-skilled group $(\mathrm{M}=0, \mathrm{SD}=0)$ (Table 2). The number of counts of camera checks during FPS gameplay were also significantly higher in the skilled $(M=7.00, S D=2.94)$ than the semi-skilled group $(M=1.40, S D=1.14)$ 
$(\mathrm{t}(3.72)=3.60, p<.05, \mathrm{~d}=2.11$ ) (Table 2). As shown in Table 2, there were no differences in visual search strategies between the two skill levels for the other items.

Table 2. Comparison of eye movement data during FPS gameplay.

\begin{tabular}{|c|c|c|c|c|c|c|}
\hline & \multicolumn{2}{|c|}{ Skilled(n=4) } & \multicolumn{2}{|c|}{ Semi-Skilled(n=5) } & \multirow{2}{*}{ t value } & \multirow{2}{*}{$95 \% \mathrm{Cl}$} \\
\hline & $M$ & SD & $M$ & SD & & \\
\hline $\mathrm{HP}$ & 2.00 & 1.83 & 1.60 & 2.51 & 0.27 & {$[-3.16,3.96]$} \\
\hline Time left & 2.75 & 2.06 & 0.60 & 1.34 & 1.90 & {$[-0.53,4.83]$} \\
\hline Lower part of the screen & 16.00 & 11.22 & 18.00 & 22.99 & -0.16 & {$[-31.93,27.93]$} \\
\hline Our team & 3.75 & 2.87 & 0.00 & 0.00 & 2.97 * & {$[0.77,6.73]$} \\
\hline Enemy team & 1.25 & 0.96 & 0.00 & 0.00 & 2.61 & {$[-0.27,2.77]$} \\
\hline Count of camera checks & 7.00 & 2.94 & 1.40 & 1.14 & 3.60 * & {$[1.15,10.06]$} \\
\hline Count of aim down sights & 26.25 & 4.03 & 19.40 & 10.33 & 1.24 & {$[-6.23,19.93]$} \\
\hline Gazing time per session & 0.30 & 0.07 & 0.27 & 0.04 & 0.93 & {$[-0.05,0.12]$} \\
\hline
\end{tabular}

Subsequently, when the averages of attention, relaxation, and zone extracted from the EEGs during FPS gameplay were compared with game skill levels, they tended to be significantly different only in terms of relaxation $(t(5.18)=2.64, p<.05, d=5.75)$.

The level of relaxation during FPS gameplay tended to be significantly higher in the skilled $(M=3.75, S D=$ 2.87 ) than the semi-skilled group ( $M=3.75, \mathrm{SD}=2.87$ ) (Figure 4).

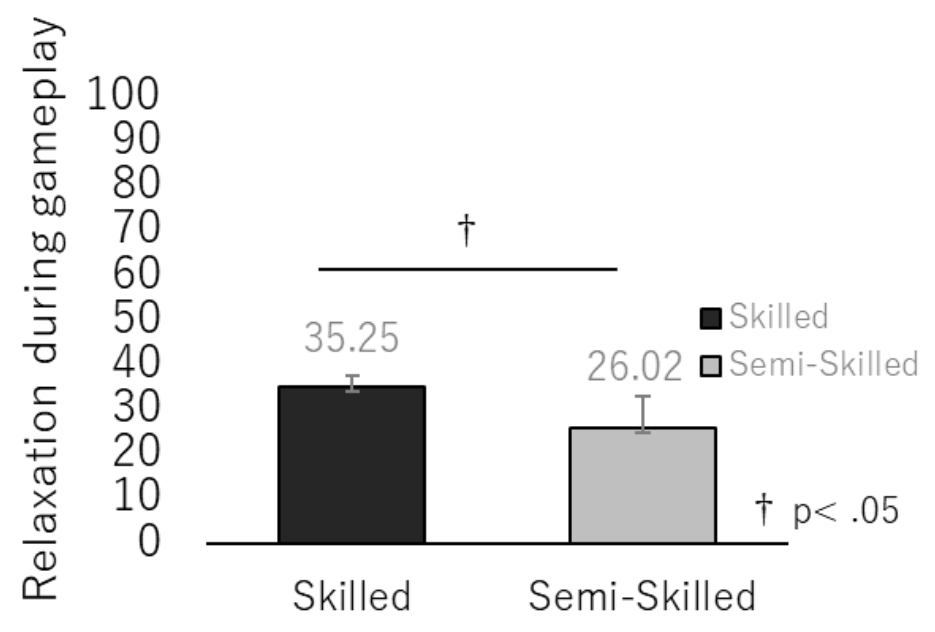

Figure 4. Comparison of the degree of relaxation during FPS gameplay between different skill levels (error bar: standard deviation) for each site.

Furthermore, a corresponding t-test that compared EEG data during the 2-minute baseline measurement and FPS gameplay found a significantly different trend only in the zone for the skilled group $(t(3)=-4.05, p$ $<.05, d=3.74)$. The degree of zone during FPS gameplay in the skilled group $(M=56.23, S D=6.62)$ tended to be significantly higher than that at baseline $(M=48.66, S D=6.44)$ (Figure 5). 


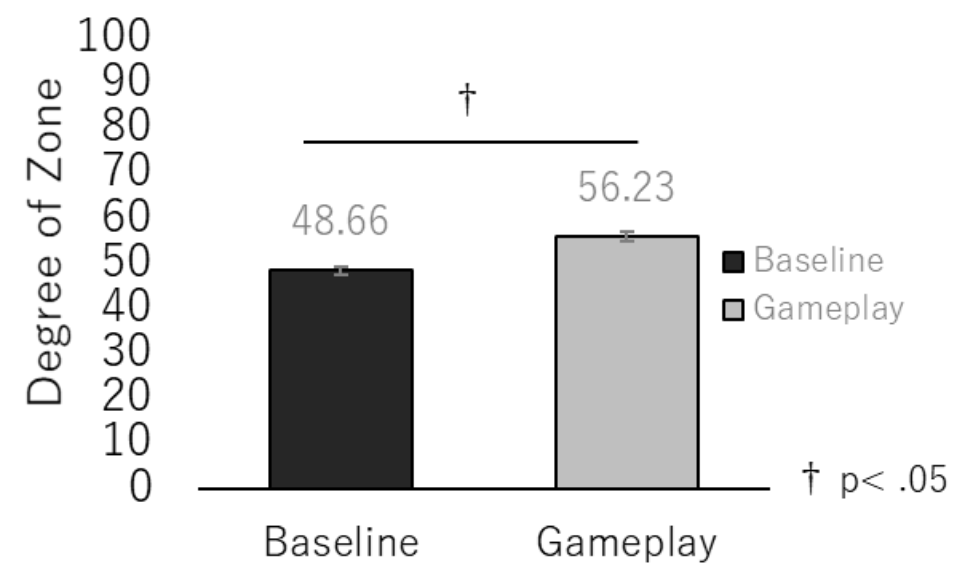

Figure 5 Comparing the average degree of zone at baseline and while playing FPS games (error bar: standard deviation) for each site.

\section{DISCUSSION}

\section{Differences in visual search strategies during FPS gameplay}

Although previous studies had rarely discussed specific visual search strategies during FPS gameplay, our $\mathrm{AOI}$ analysis showed that skilled players gazed more often at the "Our team" area fixed in the upper left corner of the monitor (refer to Figure 2), implying that the skilled group collected information about the life, death, and hit points of friendly team members about once every 60 seconds during gameplay. It is also likely that the reason why skilled groups with high game skills checked the camera more often, was to gain an advantage in the battlefield by gathering information. For example, in soccer, elite athletes with high passing accuracy have been reported to make many body and head movements (scanning) that actively and temporarily turns their faces away from the ball for gathering as much information as possible before receiving the ball (Jordet et al., 2013). Conversely, in FPS games, players can gather information by scanning the screen with their eyes without moving their heads and bodies, as in football. Therefore, it is likely that game players with higher skill levels, frequently checked the in-game camera footage to understand the enemy's position and the key points of attack and defence. It is hoped that future research will contribute to the development of research proficiency by investigating the characteristics of visual search strategies in specific situations, such as while looking at the camera, listening to enemy footsteps, etc., during FPS gameplay.

\section{Differences in EEG during FPS gameplay}

The EEG data measured in the FP1 region were converted into sensitivity values using the Sports KANSEI algorithm to compare psychological states during FPS gameplay with different levels of gaming skills. Comparisons of the means of concentration, relaxation, and zone during FPS gameplay at different game skill levels showed that only mean relaxation was significantly higher in the skilled than in the semi-skilled group. Previous studies have already shown that heart rate increases during e-sports play (Valladao et al., 2019; Sousa et al., 2020). FPS games, in particular, are a game genre where the peak heart rate can fluctuate greatly, but it is possible that skilled players may have been able to relax while playing, thereby increasing their effective field of view, and consequently improving their performance. 
In addition, the skilled group's mean sensitivity values for the zone were higher during FPS gameplay than at baseline. The zone state is a type of beta wave called the SMR wave, which is the most balanced between relaxation and concentration. In addition to SMR waves $(12-15 \mathrm{~Hz})$, there are high alpha waves $(10-12 \mathrm{~Hz})$ and low beta waves $(15-18 \mathrm{~Hz})$ in the brain waves of the mid-awake zone. If the SMR wave is the apex of the balance between relaxation and concentration; then, the more relaxed and less aroused the person is, the higher the alpha wave, whereas the more focused and more aroused the person is, the lower the beta wave. As mentioned earlier, on an average, the skilled group was more relaxed during gameplay than the semi-skilled group, suggesting that the optimal EEG band for FPS game performance can exist when the high alpha and SMR waves are in a predominant state. However, since the optimal EEG band may vary depending on the game genre, more research is needed to determine the optimal band. In addition, longitudinal experiments on the same subjects will help to improve the accuracy of the sensitivity values.

In this study, we conducted an experiment with 9 FPS game players who belonged to a vocational school and engaged in daily gameplay. However, to generalize our research findings, we need to expand the sample size and collect data from a wide range of game skill levels. First, we shall collect gold standard data from esports players who are professional athletes to allow for more detailed comparisons. There is also a need to discuss the control group's settings, as those who have never played FPS games before will need to listen to the rules first, and then practice and check the keystrokes and other aspects of the game, before they can play properly. For this reason, it is necessary to establish a common set of rules for setting the appropriate skill level for the control group.

\section{CONCLUSION}

In conclusion, this study aimed to examine the visual search strategies and EEG activities of FPS players during gameplay in e-sports. A comparison of visual search strategies during FPS gameplay based on game skill levels indicated that skilled players collected more information for situational judgment than semi-skilled players, as they gazed at fixed areas of the game screen and camera images more often than semi-skilled players. An examination of the EEG activity revealed that the skilled participants played the game in a more relaxed state than the semi-skilled ones. Furthermore, the skilled players' mean for the zone during gameplay was higher than at baseline, indicating a better balance between relaxation and concentration during FPS gameplay under psychological pressure. Future research is expected to examine the characteristics of various states during FPS gameplay.

\section{ACKNOWLEDGEMENTS}

We would like to thank Editage (www.editage.com) for English language editing.

\section{REFERENCES}

Bonny, J.W. \& Castaneda, L.M. (2017). Number processing ability is connected to longitudinal changes in multiplayer online battle arena skill. Computers in Human Behavior, 66, 377-387. https://doi.org/10.1016/j.chb.2016.10.005

Campbell, M. J., Toth, A. J., Moran, A. P., Kowal, M., \& Exton, C. (2018). eSports: a new window on neurocognitive expertise? Progress in Brain Research, 240, 161-174. https://doi.org/10.1016/bs.pbr.2018.09.006 
Furukado, R. \& Isogai, H. (2020). Differences in Visual Search Strategies Based on Multiple Object Tracking Skill Level of Soccer Athletes. Journal of Japan Society of Sports Industry, 29(2), 91-107. https://doi.org/10.5997/sposun.29.2_91

Hagiwara, G., Akiyama, D. \& Takeshita, S. (2019). Examining effectiveness of e-sports activity in Japan. Journal of Human Sport and Exercise, 14(Proc4), S1038-S1045. https://doi.org/10.14198//hse.2019.14.Proc4.66

Hagiwara, G., Akiyama, D., Furukado, R. \& Takeshita, S. (2020). A study on psychological training of eSports using digital games: Focusing on rhythm game. Journal of Human Sport and Exercise, 15(Proc3), S495-S503. https://doi.org/10.14198/ihse.2020.15.Proc3.03

Hagiwara, G., Kawahara, I. \& Kihara, S. (2020). An Attempt to Verify the Positive Effects of eSports: Focusing on Concentration and Cognitive Skill. Journal of Japan Society of Sports Industry, 30(3), 239-246. https://doi.org/10.5997/sposun.30.3_239

Hung, J.C., Lin, Z.Q., Huang, C.H. \& Lin, K.C. (2019). The Research of Applying Affective Computing Based on Deep Learning for eSports Training. In International Conference on Frontier Computing, LNEE(551), 122-129. https://doi.org/10.1007/978-981-15-3250-4_15

Jordet, G., Bloomfield, J. \& Heijmerikx J. (2013). The hidden foundation of field vision in English Premier League (EPL) soccer players. In Proceedings of the MIT sloan sports analytics conference.

Kikumasa, S. \& Kokubu, M. (2018). Decision-making and Visual Search Strategy of Baseball Catchers in a Situation of Giving Directions on a Play. Japanese journal of sport psychology, 45(1), $27-41$. https://doi.org/10.4146/jijspopsy.2017-1707

Martin-Niedecken, A.L. \& Schättin, A. (2020). Let the body'n'brain games begin: Toward innovative training approaches in esports athletes. Frontiers in psychology, 11, 138. https://doi.org/10.3389/fpsyg.2020.00138

Miyata, A., Fukui, K., Honda, K., Shigeno, H. \& Okada, K. (2004). A Proposal of Indexing Conference with Participants' Mental States. IPSJ., 45,11, 2509-2518.

Morrison, S. (2018). List of Varsity Esports Programs Spans North America. Available online at: http://www.espn.com/esports/story//id/21152905/college-esports-list-varsity-esports-programsnorth-america (accessed September 25, 2020).

Santos, A. F., Alloza, S. \& Escribano, F. (2018) Relationship between video game genres and soft skills. Available online at: https://gecon.es/game-genres-and-soft-skills/ (accessed September 25, 2020).

Söderberg, H., Khalid, J., Rayees, M., Dahlman, J., \& Falkmer, T. (2014). In video war games, are military personnel's fixation patterns different compared with those of civilians?. The Journal of Defense Modeling and Simulation, 11(4), 329-338. https://doi.org/10.1177/1548512912467867

Sousa, A., Ahmad, S. L., Hassan, T., Yuen, K., Douris, P., Zwibel, H., \& DiFrancisco-Donoghue, J. (2020). Physiological and Cognitive Functions Following a Discrete Session of Competitive Esports Gaming. Frontiers in psychology, 11, 1-6. https://doi.org/10.3389/fpsyg.2020.01030

Valladão, S., Andre, T. \& Cox, D. (2019). "Heart Rate Response During A Collegiate Esports Tournament. Journal of the American College of Sports Medicine, Suppl.51(5), 29. https://doi.org/10.1249/01.mss.0000560577.32391.44

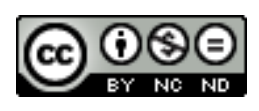

This work is licensed under a Attribution-NonCommercial-NoDerivatives 4.0 International (CC BY-NC-ND 4.0). 